\title{
ValenZa e Risorsa degli Ambienti di ApPrendimento nel Processo GLOTtodidattico
}

\author{
Giuseppe Maugeri* \\ "Proprio come non esiste un organismo senza un ambiente,così \\ non c'è un ambiente senza un organismo" \\ Lowontin, R., "The organism as the Subject and Object of \\ Evolution”, in Scientia, p. 118.
}

\begin{abstract}
Possono gli ambienti didattici essere progettati per potenziare le strategie di apprendimento degli studenti? Può lo spazio didattico avere un impatto sull'esperienza di studio di allievi adulti, influenzandone la motivazione e la performance? Sono questi alcuni degli interrogativi a cui cercheremo di dare una risposta nell'ambito di una progettazione didattica che, richiedendo un progetto analitico, include l'esperienza degli ambienti didattici come elemento strategico sia per rafforzare il processo di comunicazione tra organizzazione-docente-discenti che per l'apprendimento di questi ultimi.

PAROLE CHIAVE: psicologia ambientale; ricerca-azione; ambienti didattici; organizzazione spaziale; comfort ergonomico.

RESUMO: Os ambientes didáticos podem ser planejados para potencializar as estratégias de aprendizagem dos estudantes? O espaço didático pode ter um impacto
\end{abstract}

*Università Ca’ Foscari, Venezia. 
na experiência de estudo de alunos adultos e assim influenciar sua motivação e performance? São esses alguns dos questionamentos ao quais buscaremos responder em um planejamento didático que, exigindo um projeto analítico, inclui a experiência dos ambientes didáticos como elemento estratégico tanto para reforçar o processo de comunicação entre organização-docente-discentes quanto para a aprendizagem dos alunos.

PALAVRAS-CHAVE: psicologia ambiental; pesquisa-ação; ambientes didáticos; organização espacial; conforto ergonômico.

ABSTRACT: Can learning environments be designed to enhance the learning strategies of the students? Can the teaching space have an impact on the study experience of adult learners and, thus, influence their motivation and performance? These are some of the questions which we will seek to answer in a didactic planning that requires an analytical project, including the experience of learning environments as a strategic element both to strengthen the process of communication between organization-teacher-students, and for students' learning.

KEYWORDS: environmental psychology; action research; learning environments; spatial organization; ergonomic comfort. 


\section{Coordinate teoriche}

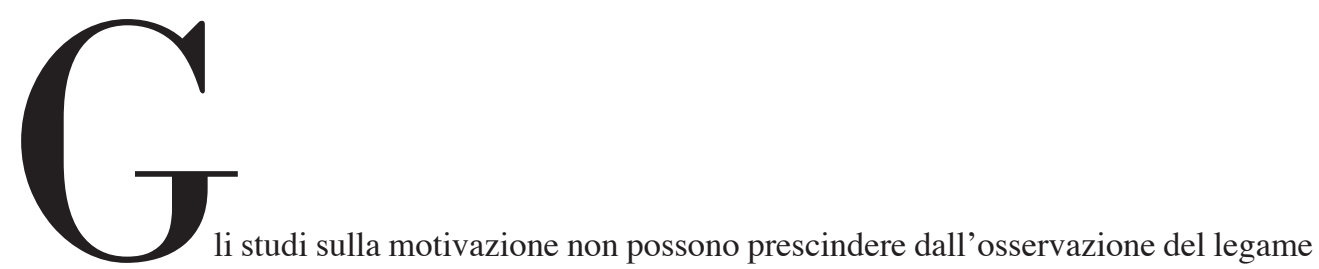

che intercorre con l'ambiente sociale (RHEINBERG, 1997) e spaziale in cui la motivazione dello studente si situa e si attua. Pertanto, questo contributo è focalizzato sulla relazione tra gli ambienti di apprendimento e gli schemi comportamentali ed emozionali dello studente inquadrati però in un'ottica di creazione di valore, ovvero di memorabilità dell'esperienza estetica, intesa come interiorizzazione di un'emozione e delle qualità sensoriali che lo studente ha percepito di un oggetto in virtù del livello di piacevolezza, di stimolazione-attivazione e di conoscenza che tale elemento ha suscitato (GOLEMAN, 1998; SCHUMANN, 2004; BALBONI, 2008). Dallo svolgimento di questi argomenti discendono aspetti e implicazioni che si richiamano alla categoria di pensiero glottodidattica della scuola veneziana. Quest'ultima ha attenzionato 
da una prospettiva umanistico-affettiva il ruolo delle emozioni dello studente nel processo di apprendimento linguistico (BALBONI, 2013); simultaneamente la scuola veneziana ha abbracciato campi disciplinari differenti al fine di elaborare un modello di educazione linguistica che, fondato sull'educabilità del sapere linguistico (FREDDI, 2010), considera gli ambienti di apprendimento come punti di apprendimento continuo, come strutture del bisogno dell'apprendente di soddisfare la categoria di bisogni secondari teorizzati da Murray (1938).

Nella misura in cui si svolge questa interrelazione tra studente-ambiente, l'indirizzo di ricerca perseguito si pone l'obiettivo secondo cui le aree didattiche vengano colte alla luce di più prospettive, vale a dire:

a. come luoghi di un sistema organizzativo e organizzato per l'apprendimento linguistico; la formulazione di un contesto organizzato costituisce un elemento decisivo per avviare pratiche di cambiamento, in presenza di determinate condizioni ambientali;

b. come spazi in cui vengono attivati processi socio-cognitivi e pratiche didattiche per lo sviluppo di abilità linguistiche. Infatti, un ambiente rilassato costruito con particolare attenzione mediante uno specifico accoppiamento cromatico, con la diffusione di fragranze aromatiche ad hoc, con la scelta di elementi dell'arredo funzionali a rendere confortevole l'esperienza di apprendimento degli studenti, agevola il discente nella comprensione e nella consapevolezza del suo bisogno di apprendimento. In casi come questo, il contesto didattico prospetta, rivela e realizza un'idea di lingua e un evento comunicativo che entrano in rapporto con le attese e le aspettative dello studente (SERRAGIOTTO, 2004, 2009);

c. come ambienti che dovrebbero maggiormente potenziare le competenze glottodidattiche del docente di lingua straniera, supportandolo nella creazione delle migliori condizioni di apprendimento. Entro il perimetro didattico si svolgono attività tali da dover consentire il movimento degli studenti e una diversa collocazione nello spazio dell'arredo. A seconda dell'impostazione tattica, al docente bisognerebbe garantire un' adeguata libertà per selezionare, 
disporre e tematizzare gli strumenti che reputa fondamentali per attivare la didattica. Perché ciò possa essere realizzato, è opportuno che il docente abbia un'idea glottodidatticamente precisa del "cosa" e del "come" voglia insegnare in classe, predisponendo un ambiente adeguato.

L'incidenza del taglio prospettico fin qui adoperato fa riferimento a modelli concettuali propri della psicologia ambientale, degli studi del marketing esperienziale ed emozionale, nonché delle scienze cognitive, rilevanti nell'attribuire al soggetto apprendente un ruolo autonomo e indipendente; assunti che si realizzano con l'elaborazione e attivazione di stimoli sensoriali, frutto della sua interazione con l'ambiente. Secondo tali presupposti, l'individuo elabora dei meccanismi di valutazione situando delle risposte differenti in base al contesto specifico, alla storia di relazioni instaurate con queste aree (BORGOGNI \& PETITTA, 2003) e alla situazionalità in cui è collocato l'evento (MC LUHAN, 1997).

Da tale ordine e funzionamento, il soggetto apprendente legittima un ruolo centrale nell'esperienza linguistica, costruendo da sé il suo progetto egodinamico e la propria conoscenza (SANTIPOLO, 2009).

Quello che prende forma è il valore di una progettualità degli ambienti finalizzata a predisporre dei luoghi pertinenti e funzionali al percorso di apprendimento dell'individuo; ciò sottende la valutazione di tutto ciò che egli fa e produce, per cui gli ambienti sono concepiti come luoghi per l'azione e l'interazione con altri individui, rafforzando in questo modo le dinamiche sociali dell'esperienza di apprendimento linguistico. I luoghi didattici, perciò, devono essere concepiti come aree preposte a ospitare una pluralità di attori che agiscono e interagiscono simultaneamente nello stesso spazio; secondo questa prospettiva, sarà fondamentale curare l'aspetto organizzativo dello spazio allo scopo di facilitare la storia di tutti i passaggi socio-cognitivi ed emotivi dello studente.

Una tale visione degli ambienti fa sì che essi non siano più una componente isolata, molecolare e ricettacolo passivo di influenze e fattori esterni al sistema, ma ne diventino parte essenziale. Infatti, l'approccio olistico con cui si classificano tali aree deputate all'apprendimento restituisce gli ambienti alla valutazione complessiva del sistema organizzativo-didattico che valuta l'adeguatezza e la pertinenza della struttura e dell'offerta formativa. Secondo questa lettura, gli ambienti di apprendimento sono elementi allineati ai valori, ai principi dell'organismo erogatore dei corsi nonché componenti soft della cultura organizzativa, giocando un ruolo specifico del contesto culturale e del sistema vitale della didattica della lingua straniera. Il 
legame fra i luoghi dell'apprendere e l'organizzazione genera una valutazione sul tipo di cultura organizzativa, dando modo all'individuo di stabilire un nesso fra la coerenza e la qualità dell'integrazione con la proposta didattica dell' istituto.

Da questi assunti si evince che la configurazione spaziale degli ambienti fa luce sia sulla tipologia di cultura organizzativa che sull'idea di conoscenza che l'organismo intende diffondere attraverso l'organizzazione e la gestione degli spazi. Quest'idea di conoscenza non può prescindere, a sua volta, dalle modalità di insegnamento della lingua straniera messe in atto dal docente, per cui nel prossimo paragrafo metteremo in rilievo l'importanza del setting fisico nel corso dei vari stadi di sviluppo dell'esperienza glottodidattica.

\section{Gli ambienti fisici nel processo glottodidattico}

Attraverso la tipologia di organizzazione spaziale dell'aula è possibile ripercorre i modelli relazionali stabiliti dai singoli approcci glottodidattici. Lo scopo è dimostrare come l'aula organizzi e disponga il progetto di azione che una particolare metodologia di insegnamento definisce con la disposizione dei banchi, ad esempio. È evidente, quindi, che alla base vi sono concezione e usi diversi dello spazio da parte dell'organismo e del docente che decide di intervenire o meno sul layout in funzione della metodologia adottata. Nel proporre una diversa distribuzione spaziale, occorre comparare due diverse filosofie di pensiero e di interpretazione dell'evento didattico in modo da designare con esattezza una precisa configurazione dello spazio in rapporto al valore assegnato al fatto linguistico e ai discenti da ciascuna delle categorie glottodidattiche esaminate.

\subsection{La visione e l'uso dell'aula negli approcci formalistici e strutturali}

Una visione dell'insegnamento di tipo "tradizionale" considera l'ambiente didattico come profondamente fondato su di un'organizzazione spaziale lineare. Essa articola un discorso silenzioso (DELEUZE, 1976), senza che vi siano variazioni delle parti e degli elementi fisici che, in una tale prospettiva di utilizzo dello spazio, assumono una posizione e un senso unico. Alla struttura scientificamente glottodidattica di tali approcci che si concentra sulle concatenazioni della lingua in atto, non considerando l'effetto che i soggetti apprendenti rilevano nella pratica di apprendimento, corrisponde una concezione inestesa dello spazio; esso pone in posizione di raccordo e in sequenza ordinata la catena dei soggetti apprendenti rispetto a ciò che essi occupano 
e producono. Ne risulta un carattere dell'ambiente didattico statico costituito dall'ordine degli apprendenti, i quali si determinano reciprocamente in lunghe file. Si tratta di una relazione spaziale e linguistica di tipo quantitativo che necessita di regolarità quali:

a. la standardizzazione e l'omogeneità di ogni componente fisico-spaziale;

b. la corrispondenza tra la metodologia di insegnamento strutturale e gli spazi didattici, elementi superflui dove mettere in scena delle strutture sempre simili, senza differenzianti e tematizzazioni;

c. la staticità nel rendere tipico e prevedibile il processo;

d. l'inespressività nel dare una forma al mondo che circonda lo studente;

e. l'inalterabilità e l'immutabilità nell'ordinare gli eventi didattici;

f. l'essenzialità nel sistema di arredo che, in linea con tale impostazione, risulta essere sbieco e superfluo.

Una visione d'insieme di questo tipo predispone un uso funzionale dell'aula per:

a. il docente che domina anche spazialmente la scena didattica. Quest'ultimo ha infatti il potere di organizzare l'aula come meglio crede, decidendo chi tra gli allievi può alzarsi, spostarsi e rispondere (dominanza verbale);

b. fare lezione con un dominio di leggi linguistiche e una struttura di interventi fissi e immutabili;

c. la memorizzazione mediante l'analisi delle categorie della lingua, la cui struttura diventa simbolica di una cultura;

d. focalizzarsi sul lavoro individuale in quanto ciò è peculiare dei movimenti singolari della LS all'interno di un contesto strutturale e cattedratico; 
Ne risulta un linguaggio spaziale frontale, rigido e vincolante, adatto a ridurre le possibilità di movimento fisico-linguistico degli studenti. A ciò è connesso un aspetto peculiare relativo alla prestazione comunicativa monodirezionale e meccanica che lo studente è chiamato a eseguire. Pertanto, lo spazio non è di tipo relazionale e dialogico, ma funge da canale e da veicolo di attività e conoscenze verbali affidate unicamente al docente.

L'esistenza di questo modello didattico giustifica la tendenza all'uniformità dei talenti e delle esperienze dei discenti grazie a un funzionamento trasmissivo del sapere (ZAMBORLIN, 2003), sviluppando quei meccanismi di memorizzazione del linguaggio utili a interpretare la nuova realtà culturale e linguistica in modo passivo, mediante una sequenza di atti prestabiliti, monocentrici, monodirezionali (BALBONI, 2008).

Una teoria di riferimento per le lingue come quella formalistica risulta adeguata a spiegare in maniera deduttiva la LS e ad analizzare soltanto la forma sintattica di una diversa coscienza in forza di comportamenti linguistici specifici. A questa linea di pensiero è collegata la riduzione in termini fisici del contesto didattico; di conseguenza, abbiamo un programma ambientale che non prevede alcun riferimento oggettuale esplicito al modello culturale della LS, mettendo in evidenza la dicotomia esistente tra:

a. categorie di pensiero statiche e il movimento di storie e interazioni che la LS risulta determinare;

b. la vita didattica della classe e la valenza significativa che quella determinata LS attribuisce al mondo dello spazio e degli oggetti.

I tools presenti in aula, dunque, non sono funzionali ad attivare una lettura di senso del processo di acquisizione. Essi coesistono come condizioni inattuali di una LS, non essendo valenze distintive del processo didattico né costituendo degli strumenti utili per facilitare l'esperienza didattica degli allievi. Lo spazio didattico, quindi, non è un aspetto considerato se non per le dimensioni che deve assumere per raccogliere il maggior numero di studenti.

In classe, la relazione fra individui e setting fisico appare adeguata nella misura in cui lo spazio supporta il ruolo e l'attività didattica del docente.

Uno schema progettuale come quello descritto mira al mantenimento di una cultura organizzativa e ambientale di natura verticale e a non generare delle distorsioni in termini di 
riconoscimento dei ruoli sociali svolti da ciascuno degli attori della didattica.

\subsection{Il ruolo del docente negli approcci formalistici e strutturali}

In classe, la relazione fra individui e setting fisico appare adeguata nella misura in cui lo spazio supporta l'attività didattica del docente e la realizzazione di questi scopi che si basano, come abbiamo detto, su un comunicativismo spicciolo.

La natura della comunicazione in classe in un approccio grammaticale-traduttivo e strutturale prevede il dominio dell'insegnante. Le forme di dominanza esercitata dal docente vengono descritte da Flanders (1970), secondo cui il docente svolge una funzione direttiva, fa lezione, occupando uno spazio verbale corrispondente in media al 70 per cento del tempo di ogni lezione (STUBBS, 1990), critica e giustifica la sua autorità, pone domande dirette. Dall'osservazione diretta della realtà di studio in Giappone presso l'IIC di Tokyo emergono ad esempio i tratti peculiari della gestione dell'intervento didattica che si pone in linea di continuità con un modello contestuale di insegnamento linguistico e un ruolo del docente che ha queste caratteristiche:

\begin{tabular}{|c|}
\hline Direttivo \\
Fa lezione \\
Dirigista e poco comunicativo \\
Critica e giustifica la sua autorità \\
Spiega tutto in maniera deduttiva \\
Dominanza verbale \\
Alta gerarchia nell'esecuzione dei compiti \\
Valori \\
Basso entusiasmo \\
Poca fiducia \\
Lavoro individuale \\
Isolamento \\
Si rivolge all'individuo \\
Negarse risorse alternative al manuale didattico \\
Demotivazione \\
dell'apprendente \\
\end{tabular}

Fig.1. Schema di riferimento didattico in un approccio grammaticale-traduttivo. Nostra libera interpretazione da Flanders. 
In un quadro di riferimento di questo tipo, il docente parla a lungo, imponendo come vere le sue idee e dando le soluzioni ai quesiti di attività didattiche del testo che si rivelano come dei veri e propri falsi pragmatici. In linea con gli studi di Balboni (2011), le annotazioni di carattere didattico sul docente affermano una dominanza di tipo:

a. strategica della "lectio", in rapporto alle mete e agli obiettivi glottodidattici con cui ha costruito la lezione;

b. semantica della discussione che si manifesta nel controllo sui temi e argomenti;

c. quantitativa, in riferimento alla durata della discussione;

d. spaziale, a causa della possibilità di scegliere come muoversi nell'aula;

e. sociale e interazionale intese come potere di costruire l'interazione e di far entrare i partecipanti.

\subsection{Il ruolo dello studente}

Non avendo una propensione relazionale né collettiva, una cornice didattica simile si dovrebbe rivolgere all'intersoggettività dell'individuo. In realtà, lo spostamento del focus sull'infrastruttura della lingua priva l'insegnamento della dimensione dell'individuo che invece cogliamo in una prospettiva umanistico-affettiva. Pertanto, lo studente assume un'identità in forza:

a. del suo posto nel banco dove egli applica per analogia al docente, per imitazione e ripetizione, le teorie del linguaggio;

b. dell'interiorizzazione di un ordine sociale che assegna una valenza simbolica alla dimensione dello spazio;

c. della sua desocializzazione. 
Lo studente assume perciò un ruolo subordinato a una struttura della comunicazione che pone il reale problema della comunicazione effettiva in lingua straniera (SERRA BORNETO, 2003). Nessuno stravolgimento e spostamento o movimento dell'individuo nello spazio; ogni soggetto e oggetto ha un ruolo predefinito e predeterminato da una struttura che, disponendo di un ordine, si attende un comportamento o un' esecuzione riconoscibile. In quest'ottica, l'apprendente è fuori dalla visione fenomenica della lingua; inoltre, la sua posizione frontale nello spazio lo priva:

a. di qualsiasi possibilità di interpretare analiticamente il luogo in cui è ospitato;

b. di attuare una strategia di osservazione ed esplorazione circa le determinanti che reputa funzionali dell'ambiente con lo scopo di sviluppare e situare in esso una sua azione cognitivo-motoria;

In base a questa prospettiva strutturale, l'ambiente è inutilizzabile per lo studente, attivando in quest'ultimo una condotta rinunciataria, obbligatoriamente passiva e marginale rispetto a ciò che l'ambiente potrebbe offrire.

\section{Nuovi paradigmi per l'apprendimento esperienziale della lingua straniera}

Il paradigma di cui ci stiamo occupando rimanda alla centralità dell'individuo in azione mediante il dispositivo ternario composto da percezione-sensazione-cognizione. Nell'universo ambientale il soggetto apprendente traduce e interpreta la sua relazione col mondo attraverso questo triplice modello unitario della vita psichica, della conoscenza del proprio corpo e della scoperta continua degli altri. Il sistema di significati ricavati costituisce l'espressione qualitativa del personale rapporto e contributo all' ambiente che diventa, in quest'ottica, un luogo dove non solo si posizionano gli oggetti animati e inanimati ma nel quale questi elementi attivano una connessione: centrale allora diventa il movimento del corpo e del pensiero tesi a stabilire una relazione di raccordo, peculiare al soggetto, con il contesto.

Secondo questa visuale, l'ambiente diventa area costruita e aggregatrice di forme di vita che qualificano la propria esperienza in virtù della qualità della relazione con lo spazio esterno 
e dell'interazione con gli altri individui. L'ambiente è dunque un fenomeno sempre aperto al mutamento e pregnante, dove il soggetto apprendente tende col pensiero alla relazione con tali spazi, costruendo delle forme e dei modelli di equilibrio. Nel fare questo, però, l'ambiente esterno compenetra, influenzandola, la dimensione creativa e vitale dell' individuo, il cui comportamento risulta essere a sua volta modellato dalle interazioni con un ambiente in costante mutamento e modificazione.

Un simile scenario permeato di una dialettica continua fra soggetti e ambienti, giustifica l'effetto psicologico e materiale che l'ambiente ha sull'apprendimento degli individui. Da qui l'importanza di valutare il modo in cui gli ambienti "entrano" nella dimensione esperienziale dello studente, enfatizzando quelle forze che determinano le scelte individuali.

Nell'ambito di un percorso di insegnamento e apprendimento delle lingue, la dimensione progettuale degli ambienti delinea una diversa sensibilità e attenzione da parte dell'ente di considerare i luoghi di studio come piattaforme in cui una pluralità di attori agisce simultaneamente entro un unico spazio; in questo modo, i confini spaziali si prestano a essere definiti dalla traduzione che gli aspetti sensoriali e fisici di tali aree generano negli individui. Per creare le migliori condizioni di apprendimento, occorre pertanto partire da un modello di aula nella quale l'allievo fa esperienza. Per questo motivo, una tale dimensione dovrebbe facilitare il discente a co-costruire la sua evoluzione in termini di miglioramento della competenza comunicativa. Ciò comporta una progettazione mirata a integrare individuo e spazio in modo che:

a. la geografia di tale luogo sia organizzata sull'apprendimento e orientata in direzione dell'apprendente; in questo modo, il contesto didattico consente di dar forma all'esperienza reale, ricomponendo gli avvenimenti e i protagonisti che hanno co-partecipato allo svolgersi dell'azione;

b. il punto $a$ prefigura uno scenario di partecipazione dello studente ai gesti e alle situazioni didattiche innescate, vedendo aumentato il suo ruolo rispetto agli obiettivi fissati.

L'ambiente costruito dallo studente è frutto anche di contrastanti percezioni del paesaggio spaziale, ma esse vanno viste in rapporto alla situazione comunicativa. Ne scaturisce una rappresentazione dell'ambiente in cui, pur essendoci delle direzioni obbligate, vi è la possibilità 
di scegliere la forma. Ne consegue una piena comprensione dello spazio, una concezione più umana e affettiva degli ambienti che ospitano l'esperienza dinamica del pensiero e dell'azione dell'individuo in relazione con gli altri.

\section{Un modello progettuale di ambiente di apprendimento "student- centred"}

L'interpretazione ricostruzionista con la quale stiamo esaminando il rapporto tra studenti e ambienti assegna perciò valore:

a. alla fisicità con cui la persona, mediante i sensi e la percezione, crea uno spazio emotivo e razionale, determinando l'ambiente in cui si trovano. Secondo una tale linea di pensiero, gli ambienti diventano parte della narrazione degli eventi in cui protagonista è lo studente e la sua capacità di comprendere e far uso dell'universo didattico che lo circonda, dirigendo la sua attività esplorativa e conoscitiva. Uno spazio, come abbiamo già analizzato, che muta con l'evolversi del suo apprendimento e che acquisita significazione nel momento dell'incontro, della comunicazione in lingua, dei risultati;

b. all'espressione cognitiva che dirige il movimento del pensiero verso l'azione e l'interazione con gli altri individui e con l'ambiente.

Queste dimensioni tengono in equilibrio l'emozione e l'attentività dello studente per cui bisogna attribuire agli ambienti una valenza progettale con lo scopo di modulare il territorio verso la sicurezza, l'affidabilità e la significatività.

Partendo da questo presupposto, attenzioneremo delle aule quattro aree di impatto: 


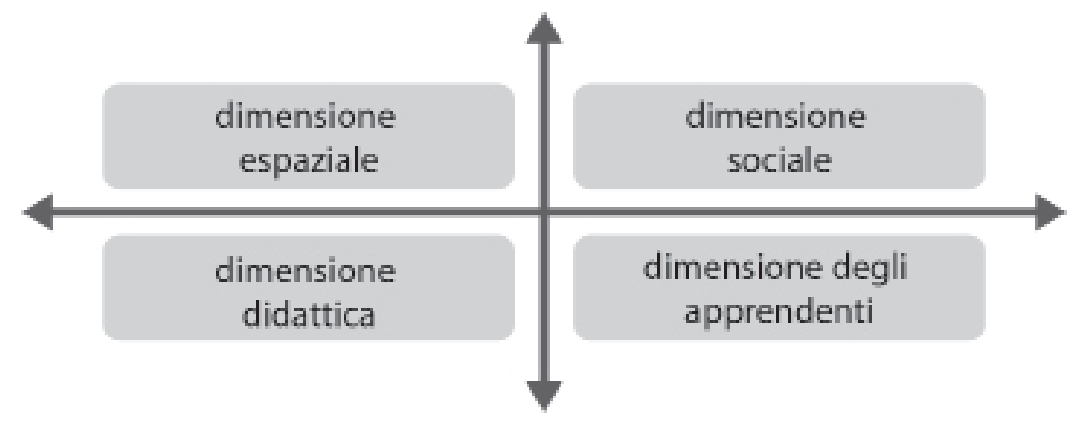

Fig. 2. Le quattro diverse aree di impatto della ricerca. Elaborazione personale.

a. la dimensione spaziale delle aule: essa tiene in stretta considerazione le componenti spaziali e strutturali, materiali e immateriali degli ambienti didattici ritenuti come una chiave del successo dell'intero corso di lingua italiana. Molte volte abbiamo assistito a forme di malessere da parte di allievi adulti nei confronti della poca accessibilità e funzionalità dell'organizzazione spaziale e fisica dei luoghi di apprendimento. Nella fattispecie, ci riferiamo ad alcune peculiarità caratterizzanti tale dimensione, ovvero il design, il colore, le attrezzature. Si tratta in realtà di elementi destinati a incidere in maniera più profonda sulla motivazione dello studente (impatto emotivo) e a implementare delle azioni di carattere cognitivo durante lo svolgimento delle attività didattiche;

b. la dimensione sociale invece si concentrerà sulla organizzazione dell'aula al fine di capire se la disposizione dei banchi, l'utilizzo dello spazio in forma tematizzata o meno, la posizione e le infrastrutture presenti, possano arrecare alcuni vantaggi per la costruzione di relazioni sociali, fluidificando in tal modo la circolazione di informazioni e scambi fra i partecipanti e valorizzando pienamente il capitale umano in una prospettiva di socializzazione continua fra studenti e corpo docente;

c. $i$ "voiced needs" dei corsisti in riferimento alla percezione che hanno a riguardo dell'ambiente, delle aspettative di miglioramento dei contesti fisici 
finalizzati a migliorare la propria esperienza all'interno di siffatti ambienti. L'obiettivo è di cogliere quei segnali forti e deboli nei confronti dell'ambiente in cui gli allievi studiano;

d. la dimensione didattica: l'ultima definizione proposta nel punto $c$ restituisce la polarizzazione del cambiamento costituito dalla coerenza e allineamento delle metodologie e dalle tecniche adottate dall'insegnante in classe con i criteri spaziali, gli obiettivi di apprendimento del corso e, non ultimo, i feedback e i bisogni degli allievi.

Si tratta di passaggi significativi e rivitalizzanti l'apprendimento delle lingue; essi possono combinarsi e ridisegnare il processo di apprendimento in funzione dei messaggi culturali che l'aula, ad esempio, è in grado di trasferire attraverso la sua descrizione e collocazione. Ma trattare l'ambiente come oggetto di studio significa rimodulare la stessa concezione dei luoghi come spazi che devono prendersi cura degli individui, facilitatori del processo di definizione delle attività in rapporto alle abilità da sviluppare. In questo senso vanno individuate tutte quelle carenze di natura infrastrutturale che possono incidere in modo negativo sulla resa didattica degli allievi. A questo aspetto si lega indissolubilmente l'effetto che tale ambiente può avere sulle risorse umane ospitate in una prospettiva di comunicazione e di sviluppo della persona; l'individuo, infatti, risponde con più potenziale agli stimoli positivi dell'aula, così come se consideriamo gli ambienti didattici dei canali di interazione, allora essi sottolineano attraverso gli elementi che ospitano, le ricadute di socializzazione dell'apprendimento nell'ambito di scenari che possono essere formali e/o informali. Ne deriva la convinzione che gli ambienti di apprendimento contribuiscono a rinforzare i legami e informalizzare lo scenario sociale dell'apprendimento. A tale risultato giungiamo attraverso un piano didattico i cui obiettivi di riferimento sono imparare a relazionarsi con gli altri e la ridefinizione, a sua volta, del modo di lavorare, nella prospettiva di un accumulo di esperienze (PRAHALAD \& RISHNAN, 2008; KOTLER, 2010) e di sviluppo di reti orizzontali (BALBONI, 2011), una considerazione che si lega con le attività impostate in classe e con le mete che vuole conseguire l'istituzione erogatrice dei corsi. Infatti, allo sviluppo e alla diffusione delle competenze si associa come obiettivo dei corsi quello di assumere consapevolezza del livello e dell'impegno della propria partecipazione a queste attività di formazione linguistica col fine di innalzare la motivazione 
ad apprendere una lingua in un contesto che permette di utilizzarla in situazione e ambienti diversi, oltre i confini dell'aula. Da questa rinnovata visuale è possibile leggere l'idea, la visione e la missione dell'organizzazione (SERRAGIOTTO \& MAUGERI, 2012) nell'elaborare dei percorsi nei quali imparare ad apprendere costituisce un radicamento profondo nella cultura dell'educazione linguistica.

\section{Il ruolo dell'istituzione per la configurazione di uno spazio innovativo per l'apprendimento}

Il compito delle istituzioni è quello di creare delle strutture adeguate per una tipologia di apprendimento che rifletta i modi di organizzare e utilizzare l'apprendimento da parte del proprio target. L'architettura fisica, in questo senso, avrà un impatto sulla natura e le modalità di apprendimento degli allievi. Come abbiamo già detto, dunque, l'organizzazione dello spazio determina:

a. il tipo di esperienza linguistica; immaginando di trovarci in un'aula tradizionale, la posizione isolata e frontale della scrivania del docente supporta un apprendimento tradizionale e di tipo trasmissivo; i banchi degli studenti disposti in lunghe file o in banchi isolati contribuisce alla predominanza dell'insegnante e a un atteggiamento di osservazione-ascolto-silenzio da parte dei discenti. Inoltre, la prestazione di tali elementi dell'arredo didattico è caratterizzata dalla mancanza di adeguati presupposti tecnici per la regolazione in base ai vari livelli di seduta degli allievi;

b. il ruolo dei partecipanti; un layout come quello tradizionale si dimostra efficace nell'attuare un modello di interazione "information-oriented", fondato sui meccanismi di input e output per la riproduzione della conoscenza. In un contesto simile, l'apprendimento è un processo individuale, di memorizzazione e di completamento di unità di contenuti che vanno sistematizzati in sequenze lineari; diversamente da questo scenario, il design di un ambiente didattico innovativo, sia a livello macro che micro, che dà spazio all'interazione e alla socializzazione dei suoi membri, presenta un contesto originale nel dare forma 
ai flussi della comunicazione mediante una disposizione circolare, con tavoli ravvicinati e mobili; un sistema circolare o a ferro di cavallo permette a tutti di imparare ad apprendere dagli altri. La maggiore prossimità dei banchi darà vita a un'esperienza di apprendimento che si giova della collaborazione fra pari e della creazione e costruzione dei saperi. In forza a tali fattori contestuali all'apprendimento, lo studente è parte di un sistema olistico il cui valore fondamentale è assegnato dalla possibilità che il singolo ha di associare ciò che fa con gli altri con la visione e la costruzione di diversi mondi. In questo senso, l'organizzazione spaziale e fisica dell'aula consente al discente di guardare e co-costruire il mondo da più punti di vista, con prestazione e un livello di impegno sempre differente;

c. la forma dell'apprendimento genera specifici linguaggi e comunità di apprendenti. I modelli di cui abbiamo sopra riferito influenzano l'esperienza di studio in un determinato contesto. In quest'ottica, a seconda del modello di spazio e di setting fisico che l'istituzione ha programmato di svolgere negli spazi, le quattro dimensioni spaziali della figura vengono formalizzate e modellizzate in modo esemplificativo con strutture di sapere e processi di insegnamento intenzionali tali da influire sul rapporto tra soggetto e oggetto e soggetto e spazio didattico.

I riferimenti menzionati pongono l'accento sulla svolta spaziale fin dai primi istanti in cui lo studente entra in contatto con gli ambienti dove si svolgerà l'apprendimento. Contrariamente agli assunti sostenuti da Chism e Bickford (2002), i quali ipotizzavano che l'apprendimento si situa soltanto nella classe e secondo un procedimento standard che accomuna la vita di una classe agli eventi che si svolgono in forma standardizzata in tutte gli altri ambienti, l'ottica socio-costruttiva approfondisce la crescita del soggetto tenendo in stretta considerazione il rapporto che il singolo stabilisce con gli altri entro un luogo che favorisce questo incontro. Le tecniche didattiche adottate seguono la trama spaziale per rielaborare l'azione degli studenti verso delle finalità di autonomia e di responsabilità. In questa direzione, lo spazio didattico diventa uno spazio di senso in cui ciascuno degli allievi cerca delle spiegazioni e trova delle risposte. Pertanto, le aule didattiche rimandano all'allievo i contenuti sui quali l'istituzione ha 
voluto contestualizzare un sapere, socializzando ogni ambiente con un linguaggio comprensibile destinato ad aumentare il fatto emotivo e a influenzare la stessa motivazione e partecipazione allo studio della lingua straniera.

\section{L'aula}

Nei successivi paragrafi descriveremo un modo per migliorare l'intero sistema ambientale affinché esso possa rispondere a determinati standard qualitativi che, pur diversificatamente percepito e individualizzato, microregoli l'attenzione e il piacere di studiare in classe.

\subsection{Relazione tra ambienti didattici e istituzionali}

Se i diversi territori interni alla scuola anticipano il progetto didattico, la dimensione fisicospaziale dell'aula contiene l'idea della conoscenza della lingua straniera che l'organismo e l'allievo hanno negoziato a partire dalla sua adesione ai corsi. Nel setting fisico e nella distribuzione delle risorse prendono forma concreta la natura e il tipo di obiettivo linguisticocomunicativo che si intende realizzare. Infatti, l'organizzazione dei banchi e la disposizione di determinati oggetti dovrebbero interpretare la visione e la tipologia di apprendimento da promuovere e altresì la modalità con cui verranno affrontati e argomentati e i contenuti didattici. La coerenza tra esterno e interno costituisce il nesso logico-percettivo-sensoriale di un legame che tende a costruire senso nel momento in cui si fa esperienza degli ambienti didattici. La natura dell'aula tende ad arricchirsi di contenuti ricomponendo o destabilizzando i fattori motivazionali e le attese dello studente ad apprendere la lingua straniera. Lo schema del sapere pertanto deve essere collegato e impostato in maniera coerente all'organizzazione degli spazi; nella teoria umanistico-affettiva, gli ambienti costituiscono lo strumento per attivare e potenziare un processo di informazione e di comprensione dell'apprendimento che si vuole perseguire. L'ambiente quindi riveste un ruolo rilevante per i seguenti motivi:

a. supporta l'esperienza di studio: lo spazio è luogo dell'evento che integra le persone con un'azione di sviluppo e di cambiamento; in tal senso bisogna privilegiare un layout in grado di stimolare i sensi evocando nell'allievo un intervento profondo, intellettuale ed emotivo, ai fini della costruzione della 
propria esperienza linguistica. Fattori tangibili quali ad esempio la distribuzione della luce, la diffusione di fragranze aromatiche ad hoc a determinate fasce di orari del giorno, la collocazione dell'elemento vegetale, hanno l'obiettivo di ridurre lo stato di ansia e di adeguare il livello di concentrazione alle richieste didattiche. A questo fine, contribuiscono gli elementi sintattici dell'aula (disposizione dei banchi, comfort delle sedie, tecnologie leggere, cablaggio assente o ridotto, ecc.) in modo che l'elemento umano sia profondamente integrato alla struttura spaziale;

b. lo schema spaziale configura un modello sociale correlato a una particolare concezione della lingua e a uno specifico approccio glottodidattico; in questa luce, sul paradigma comunicativo verte un'impostazione degli spazi finalizzata a favorire la comunicazione e lo scambio degli allievi, utilizzando nello stesso tempo tecnologie di lavoro che esplorano ed ampliano i contenuti con nuove definizioni del sapere;

c. il contesto didattico aumenta le prestazioni dello studente; un orizzonte learner centred progettato per ottimizzare l'esperienza di apprendimento consente all'allievo di

- innalzare la motivazione a stare in classe;

- accrescere la sua comprensione dello spazio;

- rappresentare

linguisticamente l'insieme delle relazioni stabilite con gli altri;

- essere

uno dei protagonisti del processo di creazione nel gruppo del sapere (SENGE, 1990).

In tal modo, l'allievo incardina nello spazio una rappresentazione e concezione dell'esperienza di tipo conoscitivo; l'incontro e l'interscambio con gli altri nei luoghi del sapere diventa occasione di formazione contestualizzata attorno a un dominio fisico-spaziale contestualizzato, personalizzato e, di conseguenza, ricreato autenticamente da dentro in rapporto alle prospettive di azione. 


\subsection{Le caratteristiche dell'aula}

L'aula dovrebbe fornire utili indicazioni allo studente riguardo al progetto didattico che si intende mettere in atto. Di conseguenza, l'ambiente andrebbe necessariamente predisposto con elementi e strumenti volti a creare impressioni e sensazioni piacevoli con lo scopo di facilitare l'esperienza di apprendimento degli allievi. Un luogo accurato e accattivante di fatto coinvolge e rende maggiormente partecipi gli allievi, determinando in essi un'attitudine e una condotta attiva, lontana da esiti di monotonia e noia (ZUCKERMAN, 1984). Nonostante le differenti caratteristiche di personalità, l'allievo costruisce una sua prima valutazione dallo schema ambientale degli spazi didattici la cui modalità di organizzazione e distribuzione rappresenta la forma della conoscenza che l'organismo vuole diffondere attraverso gli spazi. L'aula costituisce in questa cornice un microambiente che va regolato sul valore complessivo degli ambienti didattici, risultando il termine ultimo di un modello ambientale che rifletta gli schemi culturali e i comportamenti comunicativi della stessa istituzione erogatrice dei corsi. In quest'ottica, l'ambiente didattico va coordinato a tutta l'atmosfera degli altri spazi, disponendo ogni percorso con lo stesso valore e per lo stesso significato con cui l'organizzazione didattica ha promosso i corsi di lingua.

In questo senso, l'aula non è più una componente isolata ma fa parte di un contesto didattico organizzato in ogni suo punto in cui viene attivato il contatto con gli allievi. Da questo punto di vista, l'aula svolge una funzione simbolica e pratica divenendo il luogo in cui si ha la percezione e la sensazione di poter svolgere con successo un lavoro di costruzione e di sviluppo delle proprie abilità. Un ambiente rassicurante, infatti, non potrà mai essere considerato minaccioso al punto da compromettere la performance dell'allievo. Piuttosto, sarà un'area che l'allievo tenderà a controllare, sentendosi sicuro di non essere a rischio con la propria ricerca e azione.

Su questo versante, lo spazio è una componente cruciale per l'attivazione di un pensiero dinamico e per il rafforzamento dei processi motivazionali e comportamentali del discente; uno spazio circolare impostato sulla prossimità e sul contatto visivo accelererà i flussi comunicativi e gli stati di movimento. Fattori come la coesione di gruppo, l'empatia e la fiducia reciproca costituiscono una dinamica positiva per promuovere la circolazione di informazioni nell'ambiente, sfruttando il movimento circolare e rotatorio per spingere lo studente all' analisi ricorsiva dei dati, alla ridondanza dei termini con cui dare vita alla propria esperienza, resa quest'ultima più intensa grazie al confronto continuo e alla condivisione.

In un contesto simile, l'attività didattica ne risulta arricchita dalla partecipazione comunicativa 
dei vari membri, risultando impreziosita e completa dall'intervento di ciascuno che ha usufruito di un modello ambientale chiaro e orientato all'azione, finalizzato ad attribuire valore costante ad ogni persona.

\subsection{Modello di analisi ambientale dell'aula}

Per l'analisi del processo didattico in riferimento al layout degli ambienti, l'apporto strumentale e metodologico derivato dalla psicologia ambientale si rileva utile per individuare le relazioni causali prodotte fra persone e spazi e la rete di significati distribuiti nell'area dell'apprendimento di ciascuno. A tal proposito, l'analisi del contesto didattico si sviluppa in diverse fasi che sono debitrici del metodo SHEL di Edwards (1972), riadattato a fini didattici con lo scopo di intercettare la relazione tra studente e aula. In questo modo, l'analisi degli spazi interni procederà in modo da descrivere i diversi ordini spaziali relativi ai percorsi in cui transitano gli studenti.

L'intero processo può essere schematizzato nel modo seguente mediante una check list dei fattori che colgono gli ambienti didattici interni nella loro essenza:

\section{a. Ambiente interno all'organizzazione didattica:}

- distribuzione delle superfici considerate in ottica di efficienza e funzionalità alla gestione delle risorse umane impegnate nell'esperienza linguistica e formativa;

- organizzazione degli spazi in comune degli studenti (hall, scale, ascensore, bagni, front office, corridoi, sale internet, biblioteca, cinema, angolo bar, area snack, sala studio, sala studenti, spazi per privacy, area copy);

- attrezzature (wireless o cavo);

- servizi di supporto all'attività didattica (sala briefing e progettazione didattica).

\section{b. Spazi didattici:}

Aspetti relativi al comfort visivo:

- dimensione, forma e profondità dell'aula;

- illuminazione naturale ed elettrica;

- finestre apribili;

- panorama;

- colori;

- tematizzazione. 
Aspetti relativi al comfort climatico:

- ventilazione naturale;

- climatizzazione;

- personalizzazione per la gestione ad hoc della temperatura.

Aspetti relativi al comfort acustico:

- isolamento acustico;

- qualità acustica interna.

Aspetti relativi al comfort ergonomico:

- arredi (scrivanie, sedie, banchi, tavolo, poggioli, mensole, cartelloni, cattedra);

- glottotecnologie;

- configurabilità di arredi ed apparecchiature;

- personalizzazione, flessibilità ed efficienza dello spazio.

\subsection{Dati tangibili: strumenti di analisi e controllo dell'aula}

L'aula acquista una forte valenza cognitivo-emozionale in quanto è luogo del vissuto didattico dello studente. Gli elementi fisico-architettonici presenti impreziosiscono l'esperienza sensoriale dello studente e, al contempo, facilitano lo stato di benessere funzionale alla sua performance. In quest'ottica, l'aula diventa luogo di sperimentazione degli studenti in termini di flussi e relazioni fisiche, attività motorie e cognitive, attitudini e comportamenti. Nella tabella sottostante, dunque, viene tratteggiata una cornice strumentale significativa poiché le attrezzature individuate all'interno dello spazio didattico possono sostenere determinate modalità didattiche di svolgimento dell'esperienza linguistica, contribuendo alla valorizzazione di determinate competenze: 


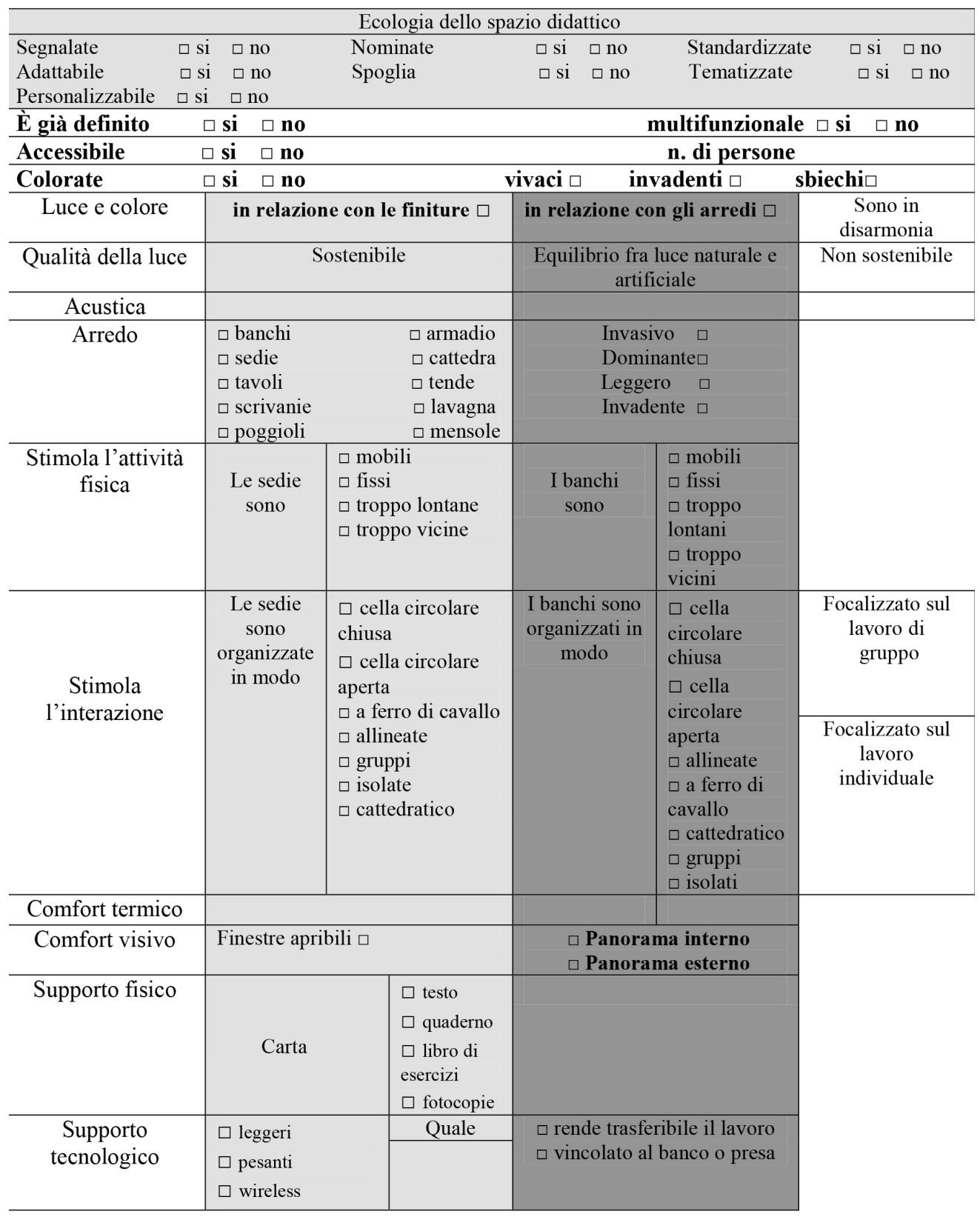

Fig.3. Ecologia dello spazio didattico. Elaborazione personale. 
L'ecologia di uno spazio didattico moderno punta a mettere nelle condizioni migliori il discente per favorire la sua concentrazione e partecipazione alle attività didattiche; l'approccio alla persona si traduce in design moderno, in spazi ampi, senza ingombri, ottimizzati per le pratiche didattiche e gli spostamenti; in una migliore resa e adattabilità dell'arredo alle peculiarità ergonomiche dei singoli, offrendo sostegno e comfort adeguato alle molteplici posizioni di seduta degli studenti. Simultaneamente, ogni aspetto tecnico si inserisce perfettamente nell'ambiente, essendo parte fondamentale di questo sistema ed elemento pratico e funzionale ai compiti che ciascuno deve svolgere dentro tale cornice; in questa direzione, i presupposti tecnici degli accessori didattici consentono allo studente di essere attivo e indipendente, nonché di utilizzare le tecnologie leggere per lo svolgimento dei vari lavori. La qualità dei fattori tangibili e intangibili (diffusione della luce, ad esempio) dovrebbe creare un movimento unico che procede nella direzione dello sviluppo dell'estetico degli spazi e verso una chiarezza visiva degli ambienti e delle aree focali. Uno scenario simile garantisce uno spazio di vita bilanciato tra comfort termico e visivo, acquisendo sussistenza nel momento in cui lo studente è consapevole di questa coerenza.

\section{Il ruolo del docente in un'aula a dimensione dello studente adulto}

Nel corso dei paragrafi precedenti abbiamo sostenuto come da una complessa struttura dello spazio fisico scaturiscano determinate pratiche di apprendimento. Questa considerazione è legata al fatto che attraverso la modalità di organizzazione dell'ambiente, un insegnante esperto è in grado di riconoscere la validità o meno di un tale modello spaziale rapportato alla tipologia di apprendimento che si vuole stabilire in classe. Pertanto dalla posizione che il docente tende ad assumere in classe e dalla modalità di organizzazione con cui lo spazio fisico si dispone ad accogliere gli allievi adulti, cogliamo la linea interpretativa che il docente riserba nei confronti della lingua da apprendere e della metodologia da sostenere per arrivare ad essa.

Continuando ad analizzare l'ambiente, da un punto di vista didattico il layout varia a seconda sia della tipologia di contesto in cui il discente deve sviluppare tali abilità, sia dall'interazione individuocontesto, oltre che da persona-persona. Da questa prospettiva, un ambiente organizzato influenza la componente percettivo-motoria delle persone ospitate, divenendo segno della realità fisica con cui interpretare l'esperienza didattica degli allievi e del loro rapporto con lo spazio; tale componente, a sua volta, esprime la tensione del soggetto apprendente nel rispondere a una spinta emotivo-interpretativa esercitata dal fattore ambientale. In tal senso, il docente dovrebbe comunicare con tutti gli elementi 
di cui dispone lo spazio, una concezione del sapere linguistico funzionale agli obiettivi dell'ambiente istituzionale che alle attese e alle motivazioni di apprendere la lingua straniera degli studenti. In quest'ottica, egli deve predisporre l'aula per concentrare-distribuire-distendere la tensione dello studente nell'ambiente. Sulla scorta di tali presupposti, ogni punto dello spazio acquista perciò valore e significato per lo studente poiché intrattiene con esso un rapporto fisico:

a. di vicinanza-prossimità-dominanza grazie al quale egli svolge in chiave contenutistica e significativa il suo ruolo nello spazio. In questo caso, il contesto determina la spinta emotiva che spinge lo studente a ricercare anche nell'ambiente la propria motivazione a fare ed apprendere;

b. di distacco fisico e psicologico per mezzo del quale egli attiva un processo di astrazione delle situazioni pragmatiche che egli vive nell'ambiente e che sperimenta durante la lezione.

Pertanto, mediante la compresenza di più elementi atti a stimolare lo studente, l'ambiente fisico influenza la performance dell'allievo in ragione della struttura organizzativa che essa ha assunto e determinato per accelerare le pratiche di studio e favorire lo stile di apprendimento degli studenti. Significativo allora come il postulato di Lewin (1946) sia secondo questa direttrice di senso rovesciata. Infatti, le forze in campo che determinano l'agire della persona $(\mathrm{P}) \mathrm{si}$ radicano nell' ambiente esterno (A) che rafforza o meno la motivazione (M).

$$
\mathrm{M}=\mathrm{P}+\mathrm{A}
$$

La tendenza dello studente a ricercare un ordine e riscontro motivazionale nell'ambiente fa sì che l'esperienza cognitiva dell'allievo non possa essere mai separata dal contesto in cui egli compie questa esperienza (BRUNER, 1996). Per questo motivo, il docente preparato glottodidatticamente concepisce l'aula come spazio di vita, strutturando dei percorsi spaziali di apprendimento nei quali utilizza tecniche didattiche che:

a. rispondono a una specifica costruzione del pensiero e a un'organizzazione dialettica tale da interpretare e accordarsi col sistema spaziale organizzato 
ad hoc;

b. agiscono per intensità sulla disposizione spaziale, divenendo in pari misura dell'ambiente, un vettore di stimolazione emotivo-cognitiva.

La valenza di tale equilibrio creato dal docente in aula rivela che la componente ambientale origina un fattore motivazionale negli studenti in ragione della qualità ambientale, della gradevolezza, del comfort dei suoi arredi - elementi architettonici che servono all'insegnante per ridurre l'ambivalenza rispetto agli obiettivi del corso e aumentare l'effetto pratico-estetico dell'esperienza didattica vissuta da ciascuno dei discenti.

\section{L'ambiente didattico in una dimensione d'uso umanistico-affettiva}

Un supporto d' aula di tipo comunicativo, contrariamente alla sequenza tradizionale descritta nel paragrafo 2, mette l'individuo nelle condizioni di far uso dell'intera personalità (WINNICOTT, 1974). Allo studente viene riconosciuto un ruolo chiave dal momento che la didattica è una risorsa sociale che deve incrementare la capacità dei discenti di osservare la lingua intesa come strumento culturalmente connotato (TORRESAN, 2014). La circolarità e la fluenza degli scambi comunicativi sono favorite da una serie di indizi spaziali che riassumiamo sinteticamente:

a. la tematizzazione dell'aula in modo da situare l'apprendimento;

b. l'aspetto progettuale dell'aula: si parte dal minimo indispensabile per poi costruire l'ambiente in funzione dei bisogni di ciascuno e della classe. La forma, in questo caso, è sempre rinnovata e in equilibrio con i fabbisogni formativi dei partecipanti;

c. l'armonia tra luce e colore;

d. la diffusione di fragranze aromatiche con lo scopo di incrementare energia e concentrazione; 
e. la presenza di elementi tattili in grado di agire in termini di qualità e comfort sul corpo dell'allievo;

f. la disposizione dell'arredo ravvicinata in modo da favorire il contatto visivo tra i partecipanti e accelerare l'analogia tra il modello didattico-input e l'elaborazione produttiva dello studente.

Si tratta di un approccio circolare alla lingua che si inserisce in una dimensione orizzontale della conoscenza. In tal senso, l'aspetto progettuale dell'aula mette in discussione l'allievo, rinnovando la sua integrazione alla cornice didattica; l'arredo è ridotto al minimo in modo da evitare aspetti inutili rispetto a ciò che richiedono le attività, salvaguardando lo spazio per i movimenti e gli spostamenti.

Un orientamento progettuale e metodologico di questo tipo mira così a recuperare la cornice comunicativa (TORRESAN, 2014), rendendo la componente fisico-percettiva una sorta di macrostrategia efficace per la comprensione di ciò che viene narrato dentro la classe. Si innesca dunque una corrispondenza tra il progetto di apprendimento e il linguaggio spaziale degli ambienti; spetterà poi al docente variare forma parallelamente alle tecniche e ai materiali proposti a lezione affinché, rendendo attrattivo e diverso lo spazio, possa valorizzare le diverse visioni sull'universo della LS.

\section{Considerazioni finali e prospettive di ricerca}

Alla luce delle considerazioni presentate, abbiamo cercato di delineare una teoria degli ambienti che riconosce in essi una delle possibili fonti maggiori di dissonanza cognitiva o, al contrario, un elemento in grado di ridurla, influenzando la motivazione e la performance degli studenti in classe. Si tratta di un fenomeno che andrebbe verificato, oltre che descritto, attraverso un'esplorazione che coinvolge tutti gli ambienti di un istituto, ente, centro linguistico al fine di far emergere nuove relazioni tra studente e spazio fisico. Le implicazioni teoriche nelle quali il soggetto tende a riferirsi all'ambiente in maniera rilevante per l'azione sottolinea un aspetto cruciale, vale a dire l'importanza di strutturare degli ambienti ad personam e secondo le caratteristiche metodologiche attivate dal docente.

Da tale vincolo di natura personale e didattica, la progettazione di nuovi contesti di 
apprendimento rappresenta certamente una nuova frontiera che occorre aprire in quanto elemento strategico e potenziale del processo di apprendimento degli studenti, tanto più se tali aree sono correlate a una stimolazione percettiva e sensoriale volta a intensificare lo slancio creativo dell'allievo, orientato verso una performance più efficace.

L'approccio teorico che abbiamo adottato lascia in sospeso numerose questioni ed evidenzia come occorra prendere in considerazione una moltitudine di variabili collegate alla geografia del contesto e alla geometria attitudinale e personale di uno specifico target esaminato; consapevoli di ciò, è indubbio però che la qualità del sistema didattico integra negli spazi il progetto dell'apprendimento dello studente, la cui portata consente di far fluire le informazioni e connettere gli individui verso un potenziale sistema di conoscenza.

In questo modo, l'organizzazione didattica amplia le occasioni di apprendimento in tutti gli ambienti in cui vi è un contatto significativo fra individui e le aree che si propongono come luoghi esperienziali e di interazione con altri soggetti.

Secondo questa visuale, l'obiettivo glottodidattico si arricchisce della riconosciuta unicità degli ambienti di studio, della prospettiva ambientale e dei suoi costrutti per dar forma alla relazione esperienziale dello studente col progettare uno spazio didattico che intensifichi la portata del bisogno di apprendere dell'allievo. In questo modo, l'istituzione e il docente trasformano un ambiente anonimo in un orizzonte preciso, ricco di vita, di piani e di intenzioni, nonché specifico alle esigenze dell'apprendente, dimostrando quindi di essere interessato in toto all'individuo. Pertanto, da qualunque angolazione li osserviamo, gli ambienti didattici si affermano come dimensione progettata e organizzata dell'esperienza di apprendimento degli individui in modo che l'area didattica risulti essere frutto di un processo continuamente verificato, negoziato e condiviso dagli studenti.

\section{Riferimenti bibliografici}

ALVESSON, M. Prospettive culturali per l'organizzazione. Roma: Guerini \& Associati, 1996.

ARGANO, L.; DALLA SEGA, P. Le nuove organizzazioni culturali. Milano: Franco Angeli, 2009.

AUGÈ, M. Non-luoghi. Milano: Eleuthera, 2005.

BALBONI, P. Conoscenze, verità, etica nell'educazione linguistica. Torino: UTET, 2012.

Elementi di Glottodidattica. Brescia: La Scuola, 1985. 
Fare educazione linguistica. Torino: UTET, 2008.

Il ruolo delle emozioni di studente e insegnante nel processo di apprendimento e insegnamento

linguistico. EL.LE, 2013, 2, 1. Disponibile in: <http://virgo.unive.it/ecf-workflow/upload pdf/ELLE 41. $p d f>$. Accesso: 21.09.2014.

Le sfide di Babele. Insegnare le lingue nelle società complesse. Torino: UTET, 2011.

BANZATO, M. Tutoring nei modelli di formazione in rete. Milano: CLUEB, 2012.

BATESON, G. Verso un'ecologia della mente. Milano: Adelphi, 1976.

BIAMONTI, A. Learning environment. Nuovi scenari per il progetto degli spazi della formazione. Milano: Franco Angeli, 2007.

BLOOM, B. Tassonomia degli obiettivi educativi. Teramo: Giunti-Lisciani, 1984.

BOCCHI, G.; VARANINI, F. Le vie della formazione. Milano: Guerini e Associati, 2013.

BORGOGNI, L.; PETITTA, L. Lo sviluppo delle persone nelle organizzazioni. Roma: Carocci, 2003.

BRUNER, J. The culture of education. Harvard: Harvard University Press, 1996.

CAON, F. Educazione linguistica e differenziazione: gestire eccellenza e difficoltà. Torino: UTET, 2008.

CAON, F.; SERRAGIOTTO, G. (eds.). Tecnologie e didattica delle lingue. Teorie Risorse Sperimentazioni. Torino: UTET, 2012.

CHISM, N.; BICKFORD, D. The importance of Physical Space in Creating Supportive Learning Environments. In New Directions for Teaching and Learning, 92, pp. 1-98, 2002.

COLBERT, F. Il marketing delle arti e della cultura. Milano: ETAS, 2009.

COONAN, C. La ricerca-azione. In LUISE, C. (ed.). Italiano Lingua Seconda. Fondamenti e metodi. Perugia: Guerra, 2003, 3, pp. 7-52.

DAMASIO, A. Emozione e coscienza. Milano: Adelphi, 2000.

DE MASI, D. (ed). L'avvento post industriale. Milano: Franco Angeli, 1993.

DELEUZE, G. Lo strutturalismo. Milano: SE, 1976.

DORNEY, Z. Conceptualizing Motivation in Foreign Language Learning. In Language Learning, 40, pp. 46-78, 1990 .

EDWARDS, E. Man and machine: Systems for safety. British Airline Pilots Associations Technical Symposium. British Airline Pilots Associations, London, pp. 21-36, 1972.

FELE, G.; PAOLETTI, I. L'interazione in classe. Bologna: Il Mulino, 2003.

FLANDERS, N. Analysing Teacher Behaviour. London: Addison Wesley, 1970.

FLETCHER, M. Teaching for Success. Folkestone: English Experience, 2000. 
FREDDI, G. Lingue: strumenti di humanitas. Studi, saggi, modelli educativi e glottodidattici, bibliografie. Milano: EduCatt., 2010.

GIBSON, J. The Ecological Approach to Visual Perception. Boston: Houghton Mifflin, 1979.

GOLEMAN, D. Lavorare con intelligenza emotiva. Milano: RCS, 1998.

GRASSILLI, B.; FABBRI, L. Didattica e metodologie qualitative. Verso una didattica narrativa. Brescia: La Scuola, 2003.

GUMPERZ, J. Contextualization and Understanding. In DURANTI, S.; GOODWIN, C. (eds.). Rethinking Context. Cambridge: Cambridge University Press, 1992, pp. 229-252.

HITCHCOCK, G.; HUGHES, D. Research and the Teacher. London: Routledge, 1989.

HOLBROOK, M.; HIRSCHMAN, E. The experiential aspects of consumption: Consumer fantasy, feelings and fun. In Journal of Consumer Research, 9, 2, pp. 132-140, 1989.

ITTEN, J. L'arte del colore. Milano: Il Saggiatore, 1961.

KNOWLES, M. The Adult Learner. A Neglected Species. Houston: Merril, 1990.

KOLB, D. Experiential learning: Experience as the source of learning and development. Englewood Cliffs (NJ): Prentice Hall, 1983.

KOTLER, P. Marketing 3.0. Milano: IlSole24Ore, 2010.

LEVY, P. L'intelligenza collettiva. Per un'antropologia del cyberspazio. Milano: Feltrinelli, 1996.

LEWIN, K. Action research and minority problems New York: Harper \& Row, 1948

MAZZOTTA, P. Gli aspetti psico-affettivi nella didattica dell'italiano come lingua straniera.In ITALS. Didattica e linguistica dell' italiano a stranieri, n. 1, pp. 47-64, 2003.

MC LUHAN, M. Gli strumenti del comunicare. Milano: Est, 1997.

MERLEAU PONTY, M. Fenomenologia della Percezione. Milano: Bompiani, 1945.

MURRAY, H. Explorations in Personality. New York: Oxford University Press, 1938.

MUZZO, P. (ed.). Internal Branding. Milano: Franco Angeli, 2010.

ORLETTI, F. La conversazione diseguale. Roma: Carocci, 2009.

PHILIPS, S. The Invisible Culture: Communication in Classroom and Community on the Warm Springs Indian Reservation. New York: Longman, 1983.

PIARDI, S.; TIEGHI, S.; NATILE, V. Office Design. Milano: Franco Angeli, 2012.

PRAHALAD, C.; RISHNAN, M. The New Age of Innovation: Driving Co-created Value through Global Networks. New York: McGraw Hill, 2008.

PUGLIESE, C. Being Creative: The Challenge of Change in the Classroom. Delta: Peaslake, 2010.

RHEINBERG, F. Psicologia della motivazione. Bologna: Il Mulino, 1997. 
ROGERS, C. On Becoming a Person. Boston: Houghton-Mifflin, 1961.

SANTIPOLO, M. (ed.). Italiano L2: dal curricolo alla classe. Perugia: Guerra, 2009.

SCHMITT, B. Experiential Marketing. New York: The Free Press, 1999.

SCHUMANN, J. The Neurobiology of Learning. Perspectives from Second Language Acquisition. Los Angeles: Erlbaum, 2004.

SEMERARO, R. La progettazione didattica. Padova: Domenghini, 2007.

SENGE, P. The fifth Discipline. New York: Currency Dubleday, 1990.

SERRA BORNETO, C. C'era una volta il metodo. Roma: Carocci, 2003.

SERRAGIOTTO, G. (ed.). Le lingue straniere nella scuola. Torino: UTET, 2004.

Dalle lingue microdisciplinari al CLIL. Torino: UTET, 2014.

Sillabo di riferimento per la formazione degli insegnanti a stranieri. Venezia: Cafoscarina, 2009.

SERRAGIOTTO, G.; MAUGERI, G. Nuove coordinate per lo sviluppo degli Istituti italiani di cultura in ambito organizzativo e didattico. In ITALS, X, 29, pp. 93-126.

SEVERINO, F. Economia e marketing per la cultura. Milano: Franco Angeli, 2011.

SOLIMA, L. L'impresa culturale. Roma: Carocci, 2005.

SOMMER, R. Personal space: The Behavioral Basis of Design. Englewood Cliffs (NJ): Prentice Hall, 1989.

STERN, G. People in context. New York: Wiley, 1970.

STUBBS, M. Language in education. In COLLINGE, N. E. (ed.). An Encyclopaedia of Language. London: Routledge, 1990, pp. 551-589.

TORRESAN, P. Intelligenze e didattica delle lingue. Bologna: EMI, 2008.

. La motivazione secondo l'Analisi Transazionale e l'insegnamento delle lingue: appunti di metologia. In Revista de Lenguas Modernas, 20, pp. 213-240, 2014.

TRIAS DE BES, F.; KOTLER, P. Innovare per vincere. Torino: Rizzoli ETAS, 2011.

VARISCO, B. Costruttivismo socio-culturale. Roma: Carocci, 2002.

WAJNRYB, R. Observation Tasks. Cambridge: C.U.P., 1992.

WEICK, K. Amendments to Organizational Theorizing. In Academy of Management Journal, 17, 3 , pp. 487-502, 1974.

. Organizzare. Milano: Isedi, 1993.

The Social Psychology of Organizing. Reading (MA): Addison Wesley, 1979.

WENGER, E. Coltivare comunità di pratica. Prospettive ed esperienze di gestione della conoscenza. 
Milano: Guerini \& Associati, 2007.

WINNICOTT, D. Gioco e realtà. Roma: Armando, 1974.

ZAGHI, K. Atmosfera e visual merchandising. Milano: Franco Angeli, 2012.

ZAMBORLIN, C. Giappone. In BALBONI, P. E.; SANTIPOLO, M. (eds.): L'italiano nel mondo. Mete e metodi dell'insegnamento dell'italiano nel mondo. Un'indagine qualitative. Roma: Bonacci, 2003, pp. 143-148.

ZUCKERMAN, M. Sensation seeking: A comparative approach to a human trait. In Behavioral and Brain Sciences, 7, pp. 413-471, 1984. 